\title{
Escarificação ácida, temperatura e luz no processo germinativo de sementes de Senna alata (L.) Roxb.
}

\author{
BRAGA, L.F. ${ }^{1 *}$; SOUSA, M.P. ; BRAGA, J.F. ${ }^{1}$; DELACHIAVE, M.E.A. ${ }^{2}$ \\ ${ }^{1}$ Universidade do Estado de Mato Grosso, Departamento de Ciências Biológicas, Rod. MT 208, Km 146, Bairro \\ Jardim Tropical, CEP: 78580-000, Alta Floresta-Brasil, *luciabraga@unemat.br ${ }^{2}$ Universidade Estadual Paulista, \\ Departamento de Botânica - Instituto de Biociências, Caixa Postal 510, CEP: 18618-000, Botucatu-Brasil \\ elena@ibb.unesp.br
}

\begin{abstract}
RESUMO: O objetivo deste trabalho foi determinar o tempo ótimo de escarificação das sementes de Senna alata em ácido sulfúrico e verificar o efeito da temperatura, em condição de luz e escuro, na germinação de sementes dessa espécie. As sementes foram imersas em ácido sulfúrico concentrado por períodos de 0,15, 30 e 60 minutos, e o teste de germinação realizado em $\mathrm{BOD}$ a $25^{\circ} \mathrm{C}$, utilizando quatro repetições de 50 sementes em cada período de tempo, sendo o delineamento estatístico inteiramente casualizado com 4 tratamentos. No segundo experimento, para verificar o efeito da temperatura e da condição de luz mais adequada a germinação, utilizouse temperaturas de 10 a $45^{\circ} \mathrm{C}$, com intervalos de $5^{\circ} \mathrm{C}$, em condição de luz fluorescente branca ou escuro contínuo (gerbox preto). Utilizou-se o delineamento experimental inteiramente casualizado, disposto em esquema fatorial 8x2, com 4 repetições, de 50 sementes cada. Em todos os testes as avaliações da porcentagem e índice de velocidade de germinação foram feitas diariamente, durante 10 dias, onde as sementes foram consideradas germinadas quando apresentaram $2 \mathrm{~mm}$ de raiz. Os dados foram submetidos à análise de variância e as médias comparadas pelo teste de Tukey a $5 \%$ de probabilidade. A escarificação do tegumento com ácido sulfúrico durante 60 minutos, foi ideal para as sementes de Senna alata, por proporcionar maiores valores na porcentagem e velocidade de germinação. A germinação ocorre entre 15 e $40^{\circ} \mathrm{C}$, sendo consideradas fotoblásticas neutras entre 20 e $40^{\circ} \mathrm{C}$ e fotoblásticas negativas preferenciais a $15^{\circ} \mathrm{C}$. O melhor desempenho germinativo foi obtido nas temperaturas de 25,30 e $35^{\circ} \mathrm{C}$, onde ocorreram maior porcentagem e velocidade de germinação.
\end{abstract}

Palavras-chave: fedegoso-gigante, germinação, dormência, regime de temperatura, fotoblastismo

\begin{abstract}
Acid scarification, temperature and light on the germination process of Senna alata (L.) Roxb. seeds. The aim of this study was to establish the optimum scarification time for Senna alata seeds in sulfuric acid, as well as to verify the effect of temperature on seed germination for this species under conditions of light and darkness. Seeds were immersed in concentrated sulfuric acid for periods of $0,15,30$ and 60 minutes, and the germination test was carried out in a $\mathrm{BOD}$ chamber at $25^{\circ} \mathrm{C}$, using four replicates of 50 seeds for each time period. Experimental design was completely randomized with 4 treatments. In the second experiment, temperatures from 10 to $45^{\circ} \mathrm{C}$, with $5^{\circ} \mathrm{C}$ intervals, were used under conditions of fluorescent white light or continuous darkness (black "gerbox") in order to verify the temperature effect and the most suitable light condition for germination. Experimental design was completely randomized, in an $8 \times 2$ factorial arrangement, with 4 replicates of 50 seeds each. In all tests, germination percentage and speed index were daily evaluated for 10 days. Seeds were considered germinated when the radicle length was $2 \mathrm{~mm}$. Data were subjected to analysis of variance and means were compared by Tukey's test at $5 \%$ significance. The tegument scarification with sulfuric acid for 60 minutes was optimal for Senna alata seeds since it led to higher values of germination percentage and speed. Germination occurs between 15 and $40^{\circ} \mathrm{C}$, and seeds are considered neutral photoblastic between 20 and $40^{\circ} \mathrm{C}$ and negative photoblastic especially at $15^{\circ} \mathrm{C}$. The best germination performance was observed at 25,30 and $35^{\circ} \mathrm{C}$, at which germination percentage and speed were higher.
\end{abstract}

Key words: "fedegoso-gigante", germination, dormancy, regime of temperature, photoblastism

Recebido para publicação em 08/02/2008

Aceito para publicação em 01/09/09

Rev. Bras. PI. Med., Botucatu, v.12, n.1, p.1-7, 2010. 


\section{INTRODUÇÃO}

Senna alata (L.) Roxb., pertencente à família Fabaceae, é planta perene arbustiva que atinge entre 1-3 m de altura, que se propaga apenas por sementes, sendo conhecida por fedegoso-gigante. É planta daninha freqüente em áreas de pastagens, beiras de estradas e terrenos baldios (Lorenzi, 2000). Com distribuição pan tropical, ocorre na América Central, América do Sul, África, Madagascar e Ásia (Missouri Botanical Garden, 2008). No Brasil ocorre desde o Norte até o Sudeste. Em alguns estados a espécie é considerada planta medicinal e de baixa palatabilidade para o gado. Em São Paulo é considerada praga em cultivos de soja, pois cresce muito rápido, provocando sombreamento das plântulas de soja (Marabesi, 2007).

A espécie é considerada medicinal possuindo propriedades antibacterianas, antifúngicas e antiinflamatórias (Abatan, 1990; Palarrichamy \& Nagarajan, 1990; Bhaskar \& Nagarajan, 1991), diurética e laxante, utilizada como matéria prima para produção de medicamentos destinados a tratar de anemia, fígado, malária e pano-branco. Apesar das propriedades terapêuticas, deve ser ministrada com cuidado, pois é também suspeita de ser tóxica e abortiva (Lorenzi, 2000).

O cultivo de espécies medicinais é dificultado muitas vezes, por que na grande maioria são espécies pouco domesticadas, não havendo conhecimento sobre a fisiologia da germinação, como ocorre com Senna alata (L.) Roxb. A germinação de espécies não domesticadas depende de fatores como superação da dormência, luz, temperatura, dentre outros.

A espécie Senna alata (L.) Roxb. apresenta tegumento impermeável dificultando a absorção de água e/ou oxigênio. Este tipo de dormência oferece resistência física ao crescimento do embrião, o que retarda a germinação. Em ambiente natural, essa dormência é quebrada por processos de escarificação, que pode ocorrer segundo VazquezYanes \& Orozco Segovia (1993) pelo aquecimento úmido ou seco do solo, por temperaturas alternadas, por microorganismos do solo ou pela ação de ácidos quando da ingestão das sementes por animais dispersores.

Em laboratório, foram desenvolvidos diversos métodos, no entanto, a eficiência desses tratamentos depende do tipo e grau de dormência que varia entre as espécies. O uso do ácido sulfúrico é comum na superação da dormência tegumentar e sua eficiência foi verificada em Senna occidentalis Link. (Delachiave \& Pinho, 2003) e Senna macranthera (Colladon) Irwin et Barneby (Santarém \& Áquila, 1995; Lemos Filho et al., 1997; Eschiapati-Ferreira \& Perez, 1997).

Além da dormência, a luz e temperatura podem interferir positiva ou negativamente na germinação das sementes. Em geral, estes fatores não têm ação independente e o efeito da luz é fortemente condicionado à temperatura, sendo a faixa de temperatura dentro da qual as sementes podem germinar é característica de cada espécie (Bewley \& Black, 1994).

Diante do exposto, foram estudados neste trabalho, o efeito de diferentes tempos de imersão em ácido sulfúrico, para superação da dormência e a influência de temperaturas e condições de luz sobre o comportamento fisiológico das sementes de Senna alata (L.) Roxb.

\section{MATERIAL E MÉTODO}

Sementes de Senna alata (L.) Roxb. (Fabaceae) foram coletadas maduras em Botucatu$\mathrm{SP}$, sendo o trabalho realizado no Laboratório de Germinação do Departamento de Botânica - IBB, UNESP, Campus de Botucatu.

Para superar a dormência do tegumento das sementes à água foi utilizado o método de imersão em ácido sulfúrico (98\% p.a.) por 0, 15, 30 e 60 minutos. Em seguida as sementes foram lavadas em água corrente por 3 minutos e passaram por assepsia com hipoclorito de sódio a $2 \%$ por 10 minutos, sendo lavadas em água destilada pelo mesmo período, quando então foram tratadas com o fungicida Benlate $500 \AA$ (Benomyl) a 0,5\% em relação ao peso das sementes.

Após cada período as sementes foram colocadas para germinar em gerbox, sobre uma folha de papel germitest umedecido com $15 \mathrm{~mL}$ de água destilada em câmara incubadora - BOD, na temperatura de $25^{\circ} \mathrm{C}$, com luz fluorescente branca contínua. O delineamento experimental utilizado foi o inteiramente casualizado, com quatro repetições, contendo 50 sementes cada.

Para verificar o efeito da temperatura e da condição de luz mais adequada a germinação, utilizaram-se temperaturas de 10 a $45^{\circ} \mathrm{C}$, com intervalos de $5^{\circ} \mathrm{C}$, em condição de luz fluorescente branca ou escuro contínuo (gerbox preto). Utilizouse $o$ delineamento experimental inteiramente casualizado, disposto em esquema fatorial $8 \times 2$, com quatro repetições, de 50 sementes cada.

As avaliações da porcentagem e índice de velocidade de germinação nos dois experimentos foram feitas diariamente, durante 10 dias, sendo calculadas de acordo com Labouriau \& Valadares (1976) e Maguire (1962), respectivamente, considerando-se germinadas as sementes que apresentaram $2 \mathrm{~mm}$ de protrusão de raiz (Rehman et al., 1996), as quais eram retiradas.

Os dados foram submetidos à análise de variância e as médias comparadas pelo teste de Tukey a $5 \%$ de probabilidade. 


\section{RESULTADO E DISCUSSÃO}

De acordo com os dados da Figura 1, observou-se que as sementes não escarificadas não apresentaram germinação, enquanto os tratamentos de imersão em ácido sulfúrico concentrado por períodos de 15, 30 e 60 minutos foram eficientes na superação da dormência das sementes de Senna alata, pois conseguiram promover a germinação, ocorrendo maiores médias de porcentagem e índice de velocidade de germinação (IVG) com o aumento do tempo de imersão.

Resultados significativos na superação da dormência com uso de ácido sulfúrico na família Fabaceae foram obtidos em diversas espécies. Nos estudos conduzidos por Cruz et al. (2007), em sementes de Schizolobium amazonicum Huber ex Ducke com diferentes tempos de imersão, o tratamento mais eficiente foi com 60 minutos. Para sementes de Bowdichia virgiloides Kunth. a porcentagem e velocidade de germinação foi beneficiada por períodos de 4, 8 e 12 minutos (Albuquerque et al., 2007). Resultados positivos também foram encontrados em sementes de Erythrina crista-galli L., com imersão por 30 minutos (Silva et al., 2006).

A escarificação do tegumento durante 60 minutos, foi ideal para as sementes de Senna alata por proporcionar $100 \%$ de germinação e maior IVG (50). Quanto à porcentagem final de germinação não houve diferença significativa entre os tratamentos de 30 e 60 minutos de imersão, porém a velocidade é estatisticamente reduzida com 30 minutos. O maior período de imersão em ácido sulfúrico proporcionou melhor remoção das partículas do tegumento (Figura 1) permitiu a embebição e favoreceu a emissão rápida da raiz primária (24 horas).

Em espécies já estudadas do gênero Senna, o ácido sulfúrico foi utilizado com eficiência para superação da dormência tegumentar por Dutra et al. (2007) em sementes de S. siamea (Lam.) H.S. Irwin \& Barneby com imersão por 15, 30 e 45 minutos, por Awodoyin \& Ogunyemi (2003) em S. obtusifolia (L.) H.S. Irwin \& Barneby durante 2 a 15 minutos, por Delachiave \& Pinho (2003) em sementes S. occidentalis Link. com imersão por 15 ou 20 minutos, por Lemos Filho et al. (1997) e Santarém \& Áquila (1995) em Senna macranthera (Colladon) Irwin \& Barneby, respectivamente por 12 e 15 minutos e por Teketay (1996) em sementes de S. bicapsularis (L.) Roxb., S. didymobotrya (Fresen.) H.S. Irwin \& Barneby, S. multiglandulosa (Jacq.) H.S. Irwin \& Barneby, S. occidentalis Link. e S. septentrionalis (Viv.) H.S. Irwin \& Barneby, durante 60 minutos.

Quando as sementes foram imersas em ácido sulfúrico em 15 e 30 minutos a germinação aos 7 dias após a semeadura foi de 50 e $93 \%$, respectivamente (Figura 1), mas o número de sementes germinadas até 24 horas foi baixo ( 2 e $34 \%$,

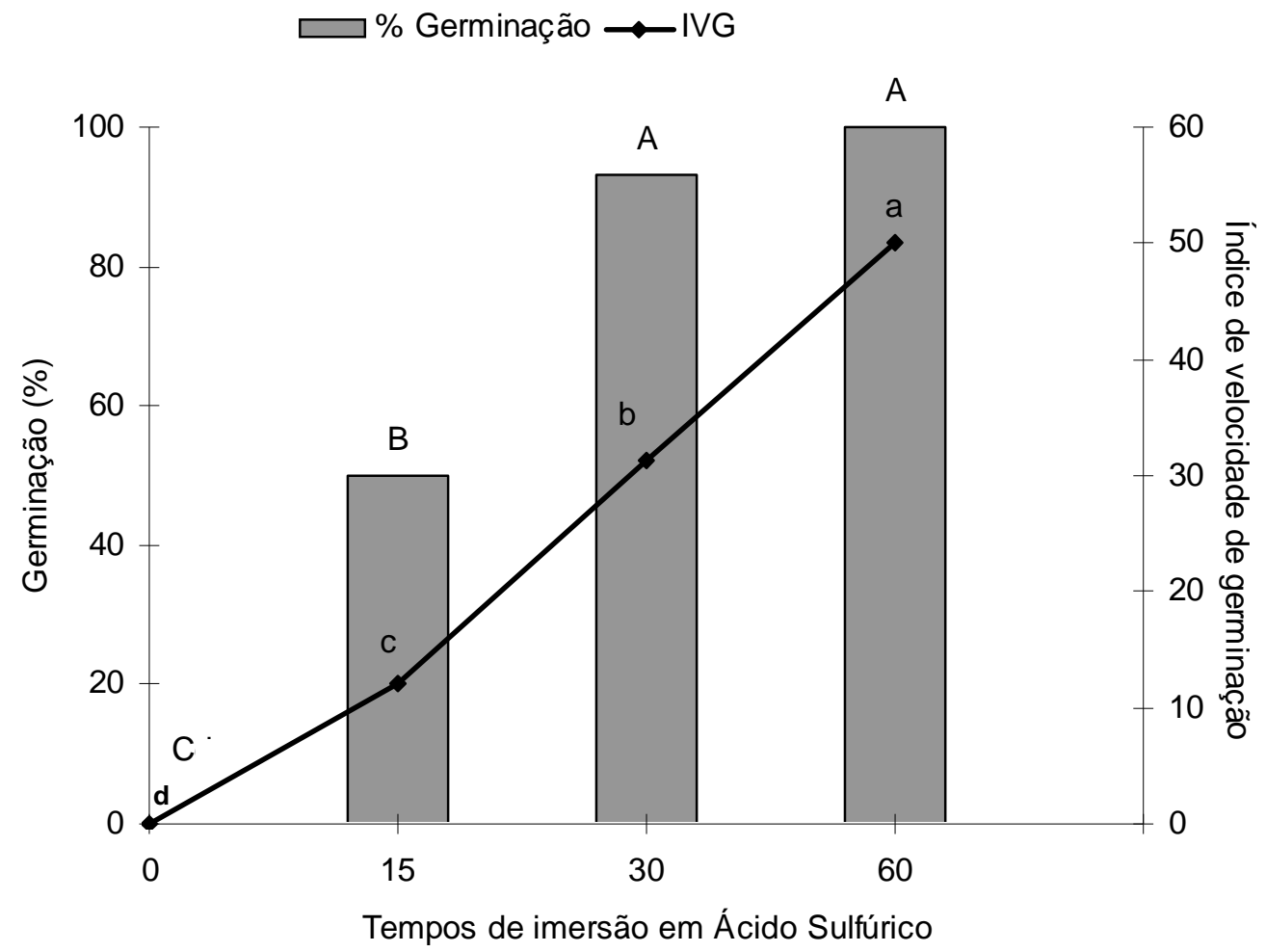

FIGURA 1. Valores médios de porcentagem e índice de velocidade de germinação (IVG) de sementes Senna alata (L.) Roxb.após imersão em ácido sulfúrico. 
respectivamente), comparando com as sementes tratadas por 60 minutos. Este comportamento pode ser explicado pela permeabilidade e desuniformidade do tamanho. Essas variabilidades na intensidade de dormência foram verificadas em sementes de Xanthium strumarium $L$., devido à posição da semente no fruto, em Halogeton glomeratus G. Mey., devido à cor (Carvalho \& Nakagawa, 2000), podendo contribuir para a distribuição temporal da germinação, com maiores vantagens na perpetuação da espécie na natureza.

As sementes de Senna alata germinaram entre 15 e $40^{\circ} \mathrm{C}$, sendo que a $15^{\circ} \mathrm{C}$ a germinação no escuro foi estatisticamente superior quando comparada à verificada na luz, na mesma temperatura. A $10 \mathrm{e}$ $45^{\circ} \mathrm{C}$ a germinação foi nula (Tabela 1 ).

A faixa de temperatura de germinação encontrada concorda com a descrição de Okusanya

TABELA 1. Porcentagem de germinação de sementes de Senna alata (L.) Roxb.sob diferentes temperaturas, na condição de luz e escuro.

\begin{tabular}{crrrr}
\hline $\begin{array}{c}\text { Temperaturas } \\
\left({ }^{\circ} \mathrm{C}\right)\end{array}$ & \multicolumn{5}{c}{$\begin{array}{c}\text { Germinação } \\
(\%)\end{array}$} \\
\cline { 2 - 5 } & \multicolumn{5}{c}{ Luz } & \multicolumn{3}{c}{ Escuro } \\
\hline 10 & 0,00 & $\mathrm{Ca}$ & 0,00 & $\mathrm{Ba}$ \\
15 & 89,00 & $\mathrm{Bb}$ & 100,00 & $\mathrm{Aa}$ \\
20 & 100,00 & $\mathrm{Aa}$ & 100,00 & $\mathrm{Aa}$ \\
25 & 100,00 & $\mathrm{Aa}$ & 100,00 & $\mathrm{Aa}$ \\
30 & 100,00 & $\mathrm{Aa}$ & 100,00 & $\mathrm{Aa}$ \\
35 & 100,00 & $\mathrm{Aa}$ & 100,00 & $\mathrm{Aa}$ \\
40 & 98,50 & $\mathrm{Aa}$ & 96,50 & $\mathrm{Aa}$ \\
45 & 0,00 & $\mathrm{Ca}$ & 0,00 & $\mathrm{Ba}$ \\
\hline CV $(\%)=2,15$ & \multicolumn{4}{c}{} \\
\hline
\end{tabular}

Médias seguidas de mesma letra, maiúscula na coluna e minúscula na linha, não diferem significativamente entre si pelo teste de Tukey, a $5 \%$.

(1978), em que sementes de espécies tropicais são tolerantes as altas temperaturas, com limite máximo igual ou superior a $35^{\circ} \mathrm{C}$ e susceptíveis a baixas temperaturas, com limite mínimo superior a $5^{\circ} \mathrm{C}$. Os resultados encontrados para temperatura mínima entre 10 e $15^{\circ} \mathrm{C}$, também foram observados em sementes de Prosopis juliflora (Sw.) DC. (Perez \& Moraes, 1990); Dalbergia nigraAllem. (Ferraz-Grande \& Takaki, 2001) e Acacia polyphylla DC. (Araújo Neto et al., 2003). Temperatura máxima entre 40 e $45^{\circ} \mathrm{C}$, também foi observada em sementes de Dalbergia nigra Allem. (Ferraz-Grande \& Takaki, 2001).

Assumindo que as sementes correspondem a um conjunto organizado de células cujo metabolismo depende essencialmente da atividade acoplada de diversas enzimas, seria esperado que, em determinadas temperaturas, a inativação de proteínas ocasionada por temperaturas extremas resultaria em um descompasso metabólico que comprometeria a germinação (Marcos Filho, 2005), isso pode, possivelmente explicar a ausência de germinação de sementes de Senna alata submetidas a 10 e $45^{\circ} \mathrm{C}$.

Segundo Bewley \& Black (1994), a temperatura afeta tanto a capacidade de germinação das sementes quanto o total de sementes germinadas, existindo limites mínimo e máximo, assim como uma faixa térmica ótima, dentro da qual se obtém a maior porcentagem de germinação. Acima e abaixo desta faixa o processo é mais lento e a porcentagem pode ser menor.

Assim, considerando o que afirmaram Bewley \& Black (1994) e observando os dados da Tabela 2, constata-se que a faixa de temperatura considerada ótima para sementes de Senna alata encontra-se entre 25 e $35^{\circ} \mathrm{C}$ na luz ou escuro contínuo, onde a velocidade de germinação e porcentagem (Tabela 1) foi máxima até 24 horas do início do experimento, comportando-se como fotoblásticas neutras.

Em espécies do gênero Senna, determinouse a faixa adequada para germinação de 20 a $25^{\circ} \mathrm{C}$ para S. bicapsularis (L.) Roxb., S. didymobotrya (Fresen.) H.S. Irwin \& Barneby, S. multiglandulosa (Jacq.) H.S. Irwin \& Barneby, S. occidentalis Link. e S. septentrionalis (Viv.) H.S. Irwin \& Barneby (Teketay, 1996), 20 a $30^{\circ} \mathrm{C}$ para Senna macranthera (Collad.) Irwin \& Barneby (Borges et al., 1997; Cassaro-Silva, 2001) e Senna occidentalis Link. (Delachiave \& Pinho, 2003) e entre 25 e 35C para Senna siamea (Lam.) H.S. Irwin \& Barneby (Dutra et al., 2007).

Considerando a temperatura de $15^{\circ} \mathrm{C}$ (Tabela 1), a mais baixa onde ocorreu germinação se verifica que apesar da elevada porcentagem de germinação na luz ou escuro contínuo (mas estatisticamente diferentes), a velocidade do processo germinativo foi menor na presença de luz (Tabela 2), indicando que sob esta temperatura a espécie se comportou como fotoblástica negativa preferencial. O contrário ocorreu nas temperaturas de 20 e $40^{\circ} \mathrm{C}$, onde a velocidade de germinação é favorecida na presença de luz (Tabela 2), porém não reduziram significativamente o total de sementes germinadas (Tabela 1).

Sementes fotossensíveis, na ausência ou presença de luz, podem ter a germinação drasticamente modificada pela temperatura (Randi \& Felippe, 1981), como ocorre em sementes de certas variedades de alface que a $25^{\circ} \mathrm{C}$ necessitam de luz para germinar, mas germinam no escuro quando em temperaturas baixas (Wareing \& Phillips, 1973), semelhante ao observado para Senna alata.

A luz exerce seus efeitos em sementes 
embebidas e apenas concentrações relativamente altas de Fvd (forma ativa), em fotoblásticas positivas, são capazes de constituir impulso para o processo de germinação, mediante a síntese de hormônios e o reinício da transcrição da mensagem genética. Nestas sementes, o Fv (forma inativa), que possui condição energética inferior, não é capaz de desencadear as reações do processo de germinação (Marcos Filho, 2005). Para Pons (1991) a temperatura tem grande efeito na razão de indução de germinação ao requerimento de luz, notando que em temperaturas baixas, o processo de germinação torna-se mais lento, como observado neste trabalho para Senna alata a $15^{\circ} \mathrm{C}$.

TABELA 2. Índice de velocidade de germinação de sementes de Senna alata (L.) Roxb.sob diferentes temperaturas, na condição de luz e escuro.

\begin{tabular}{crcrc}
\hline \multirow{2}{*}{$\begin{array}{c}\text { Temperaturas } \\
\left({ }^{\circ} \mathrm{C}\right)\end{array}$} & \multicolumn{4}{c}{ IVG } \\
\cline { 2 - 5 } 10 & 0,00 & $\mathrm{Ea}$ & 0,00 & $\mathrm{Ea}$ \\
15 & 6,16 & $\mathrm{Db}$ & 11,47 & $\mathrm{Da}$ \\
20 & 25,75 & $\mathrm{Ca}$ & 16,67 & $\mathrm{Cb}$ \\
25 & 50,00 & $\mathrm{Aa}$ & 50,00 & $\mathrm{Aa}$ \\
30 & 50,00 & $\mathrm{Aa}$ & 50,00 & $\mathrm{Aa}$ \\
35 & 50,00 & $\mathrm{Aa}$ & 50,00 & $\mathrm{Aa}$ \\
40 & 41,08 & $\mathrm{Ba}$ & 24,19 & $\mathrm{Bb}$ \\
45 & 0,00 & $\mathrm{Ea}$ & 0,00 & $\mathrm{Ea}$ \\
\hline
\end{tabular}

CV $(\%)=5,04$

Médias seguidas de mesma letra, maiúscula na coluna e minúscula na linha, não diferem significativamente entre si pelo teste de Tukey, a $5 \%$.

Dsse modo, acredita-se que, dependendo da temperatura, as sementes fotossensíveis de Senna alata apresentaram comportamento variado do processo germinativo de acordo com a fotoconversão do fitocromo que sob a temperatura de $15^{\circ} \mathrm{C}$ não requereria níveis elevados de Fve, havendo maior germinação no escuro.

Essa resposta germinativa aos diferentes ambientes de luz pode ser devida ao fato da quantidade de Fve (forma ativa do fitocromo) existente nas sementes ser suficiente para induzir a germinação (Kronenberg \& Kendrick, 1994).

Resultados semelhantes quanto à resposta fotoblástica foram verificados por Silva \& Aguiar (1998) em sementes de Ocotea catharinensis Mez., Tagetes minuta L. (Ferreira et al., 2001) e por Silva et al. (2002) em sementes de Myracrodruon urundeuva Allemão, que também foram negativas preferenciais, pois a maior porcentagem de germinação foi observada no escuro, mas apresentaram germinação na luz. Silva \& Matos (1998) classificaram as sementes de Triplaris surinamensis Cham., de fotoblásticas positivas também preferenciais, porque, apesar de germinarem em maior porcentagem na condição de luz branca, apresentaram expressiva germinação na ausência de luz.

Os resultados observados para Senna alata concordam com Carvalho \& Nakagawa (2000), que relataram que temperaturas mais baixas diminuem provavelmente a atividade metabólica das sementes, o que retarda a velocidade do processo germinativo, enquanto para Amaral \& Paulilo (1992) em temperaturas mais elevadas as velocidades de absorção da água e das reações químicas são maiores e as sementes germinam mais rapidamente. Porém, Carvalho \& Nakagawa (2000) relataram que a temperatura ótima para germinação total é diferente da ótima para índice de velocidade de germinação, o que corrobora com o resultado observado de porcentagem e velocidade de germinação de Senna alata (Tabelas 1 e 2).

Segundo Mayer \& Poljakoff-Mayber (1989), a grande dependência entre a velocidade de germinação e a temperatura é um fato estabelecido, quanto menor a temperatura menor o tempo necessário para que todas as sementes germinem e menor é a porcentagem total de germinação. A influência da temperatura na germinação é complexa, por que ela afeta cada passo do processo germinativo e depende de outros fatores.

A condição de luminosidade é um fator importante a considerar. Andrade (1995) afirmou que a indiferença à luz na germinação das sementes refere-se a um comportamento comumente descrito para árvores de sub-bosque e plantas de sombra. Entretanto, de acordo com Larcher (2000), o melhor desempenho no escuro sob qualquer temperatura, pode estar relacionado com as oscilações de temperatura e alternância de luz comumente verificadas no habitat natural da espécie, o que explicaria o comportamento observado para sementes de Senna alata em $15^{\circ} \mathrm{C}$.

\section{CONCLUSÃO}

- escarificação com ácido sulfúrico, durante 60 minutos de imersão, foi eficiente na superação da dormência tegumentar de Senna alata;

- a germinação ocorre entre 15 e $40^{\circ} \mathrm{C}$, sendo consideradas fotoblásticas neutras entre 20 e $40^{\circ} \mathrm{C}$ e fotoblásticas negativas preferenciais a $15^{\circ} \mathrm{C}$;

- melhor desempenho germinativo foi obtido nas temperaturas de 25,30 e $35^{\circ} \mathrm{C}$. 


\section{REFERÊNCIA}

ABATAN, M.O. A note on the anti-inflamatory action of plants of some Cassia species. Fitoterapia, v.61, n.4, p.336-8, 1990.

ALBUQUERQUE, K.S. et al. Métodos para a superação da dormência em sementes de sucupita-preta (Bowdichia virgilioides Kunth.). Ciência Agrotécnica, v.31, n.6, p.1716-21, 2007.

AMARAL, L.I.V.; PAULILO, M.T.F. Efeito da luz, temperatura, regulador de crescimento e nitrato de potássio na germinação de Miconia cinnamomifolia (DC.) Naudim. Insula, n.21, p.59-86, 1992.

ANDRADE, A.C.S. Efeito da luz e da temperatura na germinação de Leandra breviflora Cogn., Tibouchina benthamiana Cogn., Tibouchina grandifolia Cogn. e Tibouchina moricandiana (DC.) BAILL. (Melastomataceae). Revista Brasileira de Sementes, v.17, n.1, p.29-35, 1995. ARAUJO NETO, J.C.; AGUIAR, I.B.; FERREIRA, V.M. Efeito da temperatura e da luz na germinação de sementes de Acacia polyphylla DC. Revista Brasileira de Botânica, v.26, n.2, p.249-56, 2003.

AWODOYIN, R.O.; OGUNYEMI, S. Germination of seeds of sicklepod as affected by pre-germination treatments, fruit maturity and depth of sowing: implication in sown fallow management. Muarik Bulletin, v.6, p.44-52, 2003. BEWLEY, J.D.; BLACK, M. Seeds: physiology of development and germination. 2.ed. New York: Plenum Press, 1994. 445p.

BHASKAR, S.; NAGARAJAN, S. Actividad antibacterial de Cassia alata. Fitoterapia, v.62, n.3, p.249-52, 1991.

BORGES, E.E.L.; BORGES, R.C.G.; PAULA, N.F. Efeito da temperatura e do estresse hídrico na germinação de sementes de fedegoso (Senna macranthera (Collad.) Irwin et. Barn.) e de Leucaena leucocephala (Lam.) de Wit. Revista Brasileira de Sementes, v.19, n.2, p.156-9, 1997. CARVALHO, N.M.; NAKAGAWA, J. Sementes: ciência, tecnologia e produção. 4.ed. Jaboticabal: FUNEP, 2000. $588 p$.

CASSARO-SILVA, M. Efeito da temperatura na germinação de sementes de manduirana (Senna macranthera (Collad.) Irwin et Barn. - Caesalpiniaceae. Revista Brasileira de Sementes, v.23, n.1, p.92-9, 2001. CRUZ, E.D.; CARVALHO, J.E.U.; QUEIROZ, R.J.B. Scarification with sulphuric acid of Schizolobium amazonicum Huber ex Ducke seeds - Fabaceae. Scientia Agricola, v.64, n.3, p.308-13, 2007.

DELACHIAVE, M.E.A.; PINHO, S.Z. Scarification, temperature and light in germination of Senna occidentalis seed (Caesalpinaceae). Seed Science and Technology, v.31, n.2, p.225-30, 2003.

DUTRA, A. et al. Germinação de sementes de Senna siamea (Lam.) H.S. Irwin e Barneby - Caesalpinoideae. Revista Brasileira de Sementes, v.29, n.1, p.160-4, 2007. ESCHIAPATI-FERREIRA, M.S.; PEREZ, S.C.J.G.A. Tratamentos para superar a dormência de sementes de Senna macranthera (Collad.) Irwin et Barn. (Fabaceae-Caesalpinoideae). Revista Brasileira de Sementes, v.19, n.2, p.230-6, 1997.

FERRAZ-GRANDE, F.G.A.; TAKAKI, M. Temperature Dependent Seed Germination of Dalbergia nigra Allem (Leguminosae). Brasilian Archives of Biology and Technology, v.44, n.4, p.401-4, 2001.
FERREIRA, A.G. et al. Germinação de sementes de Asteraceae nativas do Rio Grande do Sul, Brasil. Acta Botânica Brasílica, v.15, n.2, p.231-42, 2001.

KRONENBERG, G.H.M.; KENDRICK, R.E. Photomorphogenesis in plants. Dordrecht: Martins Nijhoff, 1994. 828p.

LABOURIAU, L.G.; VALADARES, M.E.B. On the germination of seeds Calatropis procera. Anais da Academia Brasileira de Ciências, v.48, p.263-84, 1976. LARCHER, W. Ecofisiologia vegetal. São Carlos: Rima, 2000. 531p.

LEMOS-FILHO, J.P. et al. Germinação de sementes de Senna macranthera, Senna multijuga e Stryphnodendron polyphyllum. Pesquisa Agropecuária Brasileira, v.32, n.4, p.357-61, 1997.

LORENZI, H. Plantas daninhas do Brasil: terrestres, aquáticas, parasitas e tóxicas. 3.ed. Nova Odessa: Instituto Plantarum, 2000. 640p.

MAGUIRE, J.D. Speed of germination and in selection and evaluation for seedling emergence and vigour. Crop Science, v.2, n.2, p.176-7, 1962.

MARABESI, M.A. Efeito do alto $\mathrm{CO}_{2}$ no crescimento inicial e na fisiologia da fotossíntese em plântulas Senna alata (L.) Roxb. 2007. 78p. Dissertação (Mestrado) Instituto de Botânica da Secretaria de Estado do Meio Ambiente, São Paulo.

MARCOS FILHO, J. Fisiologia de sementes de plantas cultivadas. Piracicaba: Fealq, 2005. 495p.

MAYER, A.M.; POLJAKOFF-MAYBER, A. The germination of seeds. 4.ed. Oxford: Pergamon Press, 1989. 270p. MISSOURI Botanical Garden. Kemper Center for Home Gardening - Your Path to Quality Gardening Help. Plant information: alphabetical list of plants in plant finder. Disponível em: <http://www.mobot.org/gardeninghelp/ plantfinder/Plant.asp?code=A495>. Acesso em: 22 jan. 2008.

OKUSANYA, O.T. The effect of light and temperature on the germination and growth of Luffa aegyptica. Physiology Plantarum, v.44, p.429-33, 1978.

PALARRICHAMY, S.; NAGARAJAN, S. Antifungal activity of Cassia alata leaf extract. Journal of Ethnopharmacology, v.29, n.3, p.337-40, 1990.

PEREZ, S.C.J.G.A.; MORAES, J.A.P.V. Influência da temperatura, da interação temperatura-giberelina e do estresse térmico na germinação de algarobeira. Revista Brasileira de Fisiologia Vegetal, v.2, n.1, p.41-53, 1990. PONS, T.L. Induction of dormancy in seeds: its importance for the seed bank in the soil. Functional Ecology, v.5, p.669-75, 1991.

RANDI, A.M.; FELIPPE, G.M. Efeito de temperatura, luz e reguladores de crescimento na germinação de Stevia rebaudiana Bert. Ciência e Cultura, v.33, p.404-11, 1981. REHMAN, S. et al. The effect of sodium chlorilde on germination and the potassium and calcium contents of Acassia seeds. Seed Science and Technology, v.25, n.1, p.45-57, 1996.

SANTARÉM, E.R.; AQUILA, M.E.A. Influência de métodos de superação de dormência e do armazenamento na germinação de sementes de Senna macranthera (Colladon) Irwin \& Barneby (Leguminosae). Revista Brasileira de Sementes, v.17, n.2, p.205-9, 1995.

SILVA, A.; AGUIAR, I.B. Germinação de sementes de canela-preta (Ocotea catharinensis Mez. Lauraceae) sob 
diferentes condições de luz e temperatura. Revista do Instituto Florestal, v.10, n.1, p.17-22, 1998.

SILVA, A.J.C.; CARPANEZZI, A.A.; LAVORANTI, O.J. Quebra de dormência de sementes de Erythrina crista-galli. Boletim de Pesquisa Florestal, n.53, p.65-78, 2006.

SILVA, L.M.M.; MATOS, V.P. Efeito da luz e da temperatura na germinação de sementes de (Triplaris surinamensis Cham.). Revista Brasileira de Engenharia Agrícola e Ambiental, v.2, n.2, p.94-6, 1998.

SILVA, L.M.M.; RODRIGUES, T.J.D.; AGUIAR, I.B. Efeito da luz e da temperatura na germinação de sementes de aroeira (Myracrondruon urundeuva). Revista Árvore, v.26, n.6, p.691-7, 2002.
TEKETAY, D. The effect of different pré-sowing seed treatments, temperature and light on the germination of five Senna species from Ethiopia. New Forests, v.11, n.2, p.155-71, 1996.

VAZQUEZ-YANES, C.; OROZCO-SEGOVIA, A. Patterns of seed longevity and germination in the tropical rainforest. Annual Review of Ecology and Systematics, v.24, p.6997, 1993.

WAREING, P.F.; PHILLIPS, I.D.J. Dormancy. In: WAREING, P.F.; PHILLIPS, I.D.J. The control of growth and differentiation in plants. Oxford: Pergamon Press, 1973. p.223-54. 